\title{
Development and Evaluation of a Korean Version of a Thyroid-Specific Quality-of-Life Questionnaire Scale in Thyroid Cancer Patients
}

\author{
Chang Hwan Ryu, MD, PhD ${ }^{1}$ \\ Boram Park, MS² \\ Junsun Ryu, MD, PhD' \\ Youn Mi Ryu, MS ${ }^{1}$ \\ Seong Ae Jo, MS \\ You Jin Lee, MD, $P h D^{3}$ \\ Eun-Kyung Lee, MD, PhD \\ Yul Hwangbo, MD, $\mathrm{PhD}{ }^{3}$ \\ Jungnam Joo, $\mathrm{PhD}^{2}$ \\ Yuh-Seog Jung, MD, $\mathrm{PhD}$
}

\section{${ }^{1}$ Department of Otolaryngology-Head and Neck Surgery, Center for Thyroid Cancer, Research Institute and Hospital, National Cancer Center, Goyang, ${ }^{2}$ Biometrics Research Branch, Research Institute and Hospital, National Cancer Center, Goyang, ${ }^{3}$ Departement of Internal Medicine, Center for Thyroid Cancer, Research Institute and Hospital, National Cancer Center, Goyang, Korea}

\author{
Correspondence: Yuh-Seog Jung, MD, PhD \\ Department of Otolaryngology-Head and \\ Neck Surgery, Center for Thyroid Cancer, \\ Research Institute and Hospital, \\ National Cancer Center, 323 Ilsan-ro, \\ Ilsandong-gu, Goyang 10408, Korea \\ Tel: 82-31-920-1685 \\ Fax: 82-31-920-2799 \\ E-mail: jysorl@ncc.re.kr
}

Co-correspondence: Jungnam Joo, $\mathrm{PhD}$ Biometrics Research Branch, Division of Cancer Epidemiology and Prevention, Research Institute, National Cancer Center, 323 Ilsan-ro, Ilsandong-gu, Goyang 10408, Korea Tel: 82-31-920-1963

Fax: 82-31-920-2598

E-mail: jooj@ncc.re.kr

Received January 6, 2017

Accepted May 4, 2017

Published Online May 26, 2017

${ }^{*}$ Chang Hwan Ryu and Boram Park contributed equally to this work.

\section{Purpose}

The purpose of this study was to develop a Korean version of the self-reported thyroid-specific quality of life (QoL) questionnaire for thyroid cancer patients (KT-QoL), and to evaluate its reliability and validity.

\section{Materials and Methods}

Two hundred seventy-two patients who underwent thyroidectomy from January to December 2010 were recruited in this study. The original version of the thyroid QoL was translated into Korean and evaluated for its reliability and validity. Using the developed KT-QoL, the postoperative QoL was evaluated until postoperative 1 year.

\section{Results}

At the preoperative baseline, the item internal consistency (IIC) ranged from -0.19 to 0.76 , with low IIC values for items 2,17 , and 27 . Item discriminant validity ranged from $86 \%$ to $97 \%$. These values were similar at the postoperative periods. The internal consistency reliability (Cronbach's $\alpha$ ) was high for all dimensions, ranging from 0.90 to 0.95 . The test-retest reliability (intraclass correlation coefficient) was acceptable (0.74-0.82). The external validity examined by the correlation between the item $1 \mathrm{j}$ (voice changes) of KT-QoL and the voice handicap index-30 ranged from 0.51 to 0.75 . Patients' QoL scores decreased after surgery, which demonstrated the sensitivity of the questionnaire. The QoL scores in patients with lobectomy showed best QoL scores postoperatively and those with receiving radioactive iodine still showed decreased QoL scores along the postoperative periods.

\section{Conclusion}

These results demonstrate that KT-QoL is a valid instrument for evaluating QoL of Korean patients with thyroid cancer.

\section{Key words}

Quality of life, Thyroid gland, Thyroid neoplasms, Surveys and questionnaires, Validation studies 


\section{Introduction}

Traditionally, the aim of cancer treatment has been focused on prolonging patient survival. However, in the case of thyroid cancer, differentiated thyroid carcinoma (DTC), which comprises approximately $90 \%$ of all thyroid malignancies, shows good survival outcomes [1]. Surgery is a standard strategy for the treatment of thyroid cancer, and adjuvant radioactive iodine (RAI) therapy is often administrated in most advanced cases [2,3]. The average life-expectancy of thyroid cancer patients is not different from that of the general population $[4,5]$. Therefore, the current thyroid cancer guidelines for patient care are shifting toward decreasing morbidity and increasing the quality of life (QoL) from merely increasing the survival rate $[2,3]$.

Despite the recent introduction of robotic/endoscopicassisted thyroidectomy and intraoperative neuromonitoring, which have resulted in improved cosmetic and atraumatic surgical outcomes, most thyroid cancer survivors experience the surgery-related complications including recurrent laryngeal nerve palsy and / or hypocalcemia [6,7]. RAI therapy has several complications including salivary, eye, gastrointestinal, and hematologic disturbances, depending on the total dose of administered RAI [8]. Also, thyroid cancer survivors are exposed to the high rate of recurrence and the recurrent tumors might develop 10-20 years after the initial treatment [9]. In cases of these recurrent tumors, additional surgery and RAI therapy are indicated. Meanwhile, psychologically, thyroid cancer survivors might have adaptation problems [10]. All these could potentially affect patient's health-related QoL.

Several questionnaires exist to measure patient's healthrelated QoL in thyroid cancer. The most commonly used measures include short-form 36 (SF-36), European Organization for Research and Treatment in Cancer-Quality of Life Questionnaire C30 (EORTC-QLQ C30), Functional assessment in cancer therapy-general (FACT-G), and thyroid-specific QoL scale (T-QoL) [10]. The first three questionnaires were developed to measure the QoL in general cancer survivors, and all of these were developed and validated in the Korean population [11-13]. As mentioned earlier, thyroid cancer survivors often face unique QoL problems, as compared to survivors of other types of cancer. Therefore, it is important to focus on thyroid-specific QoL issues to investigate the impact of primary treatment on QoL, which can be used to devise follow-up strategies. T-QoL was developed in the United States, in English, to focus on the health-related QoL specific to thyroid cancer survivors [14].

No QoL questionnaires designed specifically for thyroid cancer patients have undergone systematic development and evaluation in Korea. A simple and direct translation of the published questionnaire usually fails to convey the originally intended contents because of linguistic and cultural differences. Thus, more systematic translation and cross-cultural adaptation processes are essential. Therefore, we conducted this study to develop a Korean version of the self-reported questionnaire for thyroid cancer patients (KT-QoL), and to evaluate its reliability and validity.

\section{Materials and Methods}

\section{Korean translation}

The English version of the T-QoL was translated into Korean by two bilingual translators. Physicians reviewed the initially produced Korean version, which was then backtranslated into English by a bilingual physician. Both English versions were compared for consistency in order to detect the presence of ambiguous or inadequate items, and then were used to produce the final version (Supplementary Material).

\section{Participants}

Patients planning to undergo thyroidectomy were recruited between January and December 2010 with the following criteria: (1) age 25-80 years, (2) diagnosed with differentiated thyroid carcinoma, and (3) no previous cancer history. Based on these criteria, 272 patients were eligible for inclusion in the analyses. All participants underwent thyroidectomy as described previously [15]. The surgical procedures were performed by experienced thyroid surgeons. Prophylactic or therapeutic central neck dissection, which included the pretracheal, prelaryngeal, and paratracheal nodes, was performed on all patients. RAI therapy was recommended 2-4 months after surgery, depending on the risk stratifications included in the guidelines of the American Thyroid Association, after a full interdisciplinary discussion [16]. All enrolled patients were followed for $>12$ months. KT-QoL and Voice Handicap Index 30 (VHI-30) were taken preoperatively and at 1 month, 6 months, and 12 months after surgery. All participants provided a written informed consent according to the policies and procedures approved by the institutional review board of the National Cancer Center, Korea (NCCNCS-09-294).

\section{Survey questionnaire details}

The T-QoL contains 30-items, ordinal disease-specific scales with four dimensions: physical (items 1-2), psycholog- 
ical (items 3-15), social (items 16-23), and spiritual (items 2430) well-being. However, some of items (items 1, 12, 15, 20, and 21) have multiple sub-scale items. We dealt with each of these items as separate individual items. Each item was scored on a 10-point scale, with 0 representing the worst possible and 10 representing the best possible QoL. The scores were reversed for negatively worded items (item 1, 3, 10-23, and 27), and hence a lower score indicated a lower QoL. Because item 12 (12a-g) could only be answered after surgery, it was excluded from the analysis. Patients with missing data on more than $10 \%$ of the items were excluded from the analysis, and the remaining missing data were imputed using the regression method [17].

\section{Statistical analysis}

Demographic and clinical characteristics of the study population are presented as median (range) for continuous, and counts (proportions) for categorical variables. The multi-item scale score for each dimension was calculated by summing the scores, and then by transforming scores to a scale of 0 to 100. The QoL scale score for each dimension is then summarized as a median scale score (interquartile range) and the proportion of respondents scoring at the highest (ceiling) and lowest (floor) level. Each item's internal consistency (IIC) was calculated by the Pearson's correlation between an item and the scale score from all the remaining items in the same dimension [18]. Moreover, item discriminant validity (IDV) was measured by examining whether an item's correlation with its hypothesized dimension is significantly higher than that with other dimensions. The internal consistency reliabilities of items in each dimension and the whole questionnaire were evaluated by Cronbach's alpha coefficient. To assess test-retest reliability, the intraclass correlation coefficient (ICC) between two time points was measured for 1 month, 6 months, and 12 months after surgery. The external validity of the questionnaire was examined by the Spearman's correlation coefficient between the item $1 j$ (voice changes) of KT-QoL and VHI-30. The Spearman's correlation coefficients between scale scores of different dimensions were calculated to investigate the relationship between dimensions. The mean scale scores between different time points and clinical groups (sex, treatment modality, and tumor stage) were compared based on a mixed effect model. All the statistical analyses were performed using R statistical software ver. 3.1.2 and SAS ver. 9.3 (SAS Institute Inc., Cary, NC).
Table 1. Demographic and clinical characteristics of the study population

\begin{tabular}{lc} 
Variable & No. $(\%)(\mathbf{n}=\mathbf{2 5 9})$ \\
\hline Age, median (range, yr) & $47(19-75)$ \\
\hline Sex & $58(22.4)$ \\
\hline Male & $201(77.6)$ \\
\hline Female & $159(61.4)$ \\
\hline TNM stage (AJCC 7th) & $95(36.7)$ \\
\hline Stage I, II & $5(1.9)$ \\
\hline Stage III, IV & $59(22.8)$ \\
\hline Benign & $56(21.6)$ \\
\hline Type of surgery & $144(55.6)$ \\
\hline Lobectomy & \\
\hline Total thyroidectomy & \\
\hline Total thyroidectomy+RAI & \\
\hline AJCC, American Joint Committee on Cancer; RAI, radioac- \\
tive iodine.
\end{tabular}

\section{Results}

\section{Baseline characteristics}

A total of 272 thyroid cancer patients were recruited in the study. After excluding patients with missing data on more than $10 \%$ of the items, data on 259 patients were used for the analysis of the preoperative QoL, and 237, 233, and 223 patients were used for 1 months, 6 months, and 12 months after surgery, respectively. The median age of the study population at baseline was 47 years (range, 19 to 75 years), and 201 of the participants $(77.6 \%)$ were female. Within the study population, 201 patients $(77.6 \%)$ underwent total thyroidectomy and of them, 144 patients (55.6\%) administered RAI 2-4 months after surgery. Overall stage III and IV were 95 patients (36.7\%) (Table 1). Among the 259 subjects, no patient had a T4 level $<0.89 \mathrm{mg} / \mathrm{mL}$ or a thyroid-stimulating hormone level $>4.05 \mu \mathrm{IU} / \mathrm{mL}$ at any time point (before and 1, 6, and 12 months after thyroidectomy).

\section{Reliability and validity of the KT-QoL}

The item internal validity was confirmed by the acceptable IIC and IDV values. At baseline (preoperative), the IIC values ranged from -0.19 to 0.76 . Low IIC values were obtained from items 2,17, and 27, which correspond to the overall physical well-being, support on social concern, and uncertainty on spiritual well-being, respectively. The IICs of all the items except these three were greater than 0.4 at all the time points. The IDV ranged from $86 \%$ to $100 \%$, which represents excellent discrim- 


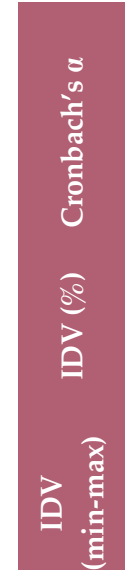

ஓ.

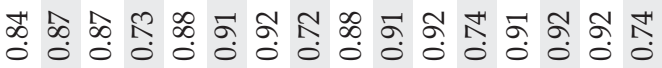

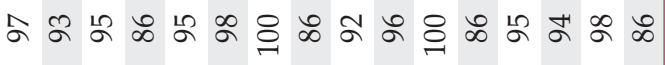

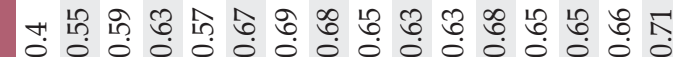

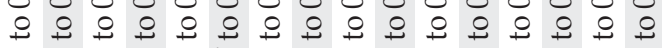
$\exists$ ㄴำ

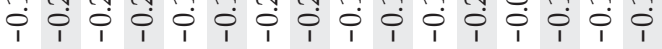

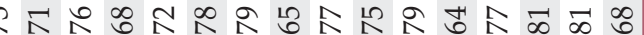
$\mathcal{U}=1$

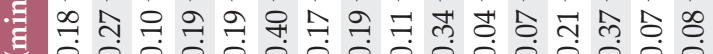

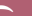

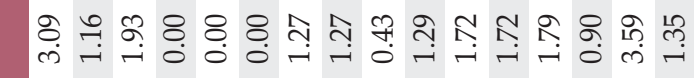

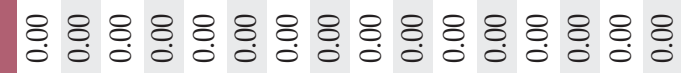

๘ नूर 우웅ㅇㅇㅇㅇㅇㅇㅇㅇㅇㅇㅇㅇㅇㅇㅇㅇㅇㅇㅇㅇㅇㅇ 운

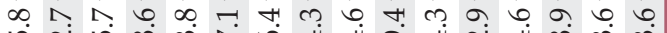

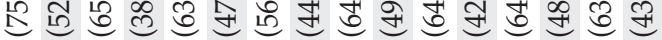

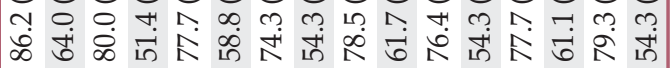
तुँ :

范

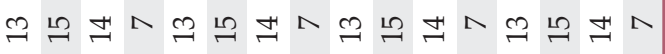

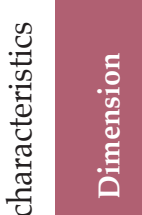
0
0
0
0
0
0
0
0
0
0
0
0
0
0
0
0
0
0
0

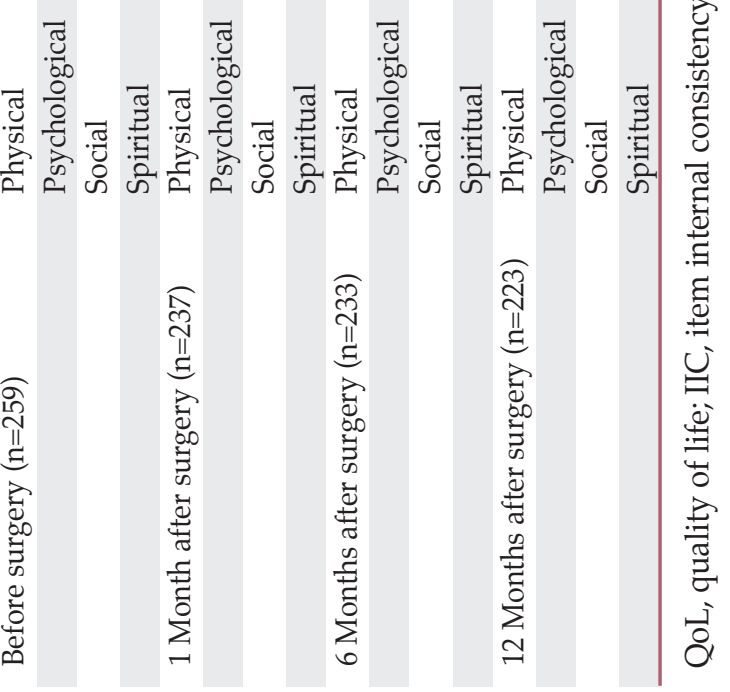


Table 3. Test-retest ICC between three tests ( 1 month, 6 months, and 12 months after surgery) for reproducibility

\begin{tabular}{|c|c|c|c|}
\hline \multirow[b]{2}{*}{ ICC between $1 \mathrm{mo}$ and $6 \mathrm{mo}(\mathrm{n}=210)$} & Dimension & \multicolumn{2}{|c|}{ ICC } \\
\hline & Physical & 0.716 & 0.813 \\
\hline & Psychological & 0.748 & \\
\hline & Social & 0.727 & \\
\hline & Spiritual & 0.756 & \\
\hline \multirow[t]{4}{*}{ ICC between $1 \mathrm{mo}$ and $12 \mathrm{mo}(\mathrm{n}=207)$} & Physical & 0.701 & 0.742 \\
\hline & Psychological & 0.681 & \\
\hline & Social & 0.655 & \\
\hline & Spiritual & 0.694 & \\
\hline \multirow[t]{4}{*}{ ICC between 6 mo and $12 \mathrm{mo}(\mathrm{n}=208)$} & Physical & 0.799 & 0.822 \\
\hline & Psychological & 0.750 & \\
\hline & Social & 0.719 & \\
\hline & Spiritual & 0.757 & \\
\hline
\end{tabular}

ICC, intraclass correlation coefficient.

ination between dimensions. The lowest value of IDV was observed for the spiritual domain, which was $86 \%$ (Table 2).

The percentage of the patients with highest and lowest possible scores was low, evidenced by the flooring and ceiling percentages presented in Table 2. The internal consistency reliability was high, as verified by Cronbach's $\alpha$ coefficients ranging from 0.90 to 0.95 at all the time points. Cronbach's $\alpha$ coefficient of each dimension ranged from 0.72 to 0.92 , with lowest value observed for the spiritual dimension (Table 2). The test-retest reliability was examined using ICC between postoperative 1 month and 6 months, 1 month and 12 months, and 6 months and 12 months. The ICCs between postoperative 1 and 6 months, and 6 and 12 months, were 0.81 and 0.82 , respectively. The value was reduced to 0.74 between 1 and 12 months (Table 3).

To examine the external validity, the Spearman's correlation between item $1 \mathrm{j}$ (voice changes) of KT-QoL and VHI-30 was calculated. The Spearman's correlation at each time point ranged from 0.51 to 0.75 ( $p<0.001$ ), which was the strongest at 1 month after surgery, followed by 12 months after surgery, 6 months after surgery, and the preoperative period (Table 4). All dimensions except the spiritual dimension were well correlated with each other, with correlations range from 0.37 to 0.91 (Table 5).

\section{Comparison of mean KT-QoL scale scores over time}

The total QoL score decreased significantly after surgery at all the time points. After a rapid decrease at postoperative 1 month, it increases at 6 and 12 months postsurgery, but not fully to the level of the preoperative baseline $(p<0.001)$. The scores for physical well-being decreased sharply at 1 month postsurgery, and remained low until 12 months postsurgery.
Table 4. Spearman's correlation coefficients between KT-QoL $1 j$ item and VHI-30

\begin{tabular}{lcc} 
& Correlation & p-value \\
Before surgery & 0.510 & $<0.001$ \\
1 Month after surgery & 0.750 & $<0.001$ \\
6 Months after surgery & 0.603 & $<0.001$ \\
12 Months after surgery & 0.622 & $<0.001$ \\
\hline
\end{tabular}

KT-QoL 1j item: voice changes. KT-QoL, Korean version of the self-reported questionnaire for thyroid cancer patients; VHI-30, Voice Handicap Index 30.

As for the QoL scores for psychological and social well-being, they also decreased significantly at 1 month postsurgery. However, these scores were recovered fully at 6 and 12 months postsurgery. On the other hand, the scores for the spiritual dimension did not change substantially after surgery $(\mathrm{p}=0.355)$ (Fig. 1).

The total QoL scores stratified by surgical extent and postoperative RAI therapy were significantly different along the postoperative periods $(p=0.001)$. The total QoL scores in patients with lobectomy showed best QoL scores postoperatively. Patients with total thyroidectomy groups experienced decreased QoL at postoperative 1 month. These scores were recovered at postoperative 6 months and 12 months for patients who did not receive RAI. However, the consistently decrease QoL scores were observed for those who received RAI. As for the QoL scores for psychological $(p=0.043)$ and social well-being $(p=0.019)$, similar trends with overall QoL sores were observed. The scores for physical and spiritual well-being were not significantly different between three 
Table 5. Spearman's correlation coefficients between KT-QoL dimensions

\begin{tabular}{|c|c|c|c|c|c|}
\hline & Physical & Psychological & Social & Spiritual & Total score \\
\hline \multicolumn{6}{|l|}{ Before surgery } \\
\hline Physical & 1.000 & & & & \\
\hline Psychological & 0.490 & 1.000 & & & \\
\hline Social & 0.373 & 0.603 & 1.000 & & \\
\hline Spiritual & -0.044 & 0.030 & -0.018 & 1.000 & \\
\hline Total score & 0.662 & 0.856 & 0.784 & 0.250 & 1.000 \\
\hline \multicolumn{6}{|c|}{1 Month after surgery } \\
\hline Physical & 1.000 & & & & \\
\hline Psychological & 0.596 & 1.000 & & & \\
\hline Social & 0.689 & 0.687 & 1.000 & & \\
\hline Spiritual & -0.082 & 0.108 & -0.102 & 1.000 & \\
\hline Total score & 0.799 & 0.896 & 0.857 & 0.177 & 1.000 \\
\hline \multicolumn{6}{|c|}{6 Months after surgery } \\
\hline Physical & 1.000 & & & & \\
\hline Psychological & 0.626 & 1.000 & & & \\
\hline Social & 0.688 & 0.666 & 1.000 & & \\
\hline Spiritual & 0.012 & 0.150 & 0.003 & 1.000 & \\
\hline Total score & 0.820 & 0.894 & 0.835 & 0.275 & 1.000 \\
\hline \multicolumn{6}{|c|}{12 Months after surgery } \\
\hline Physical & 1.000 & & & & \\
\hline Psychological & 0.654 & 1.000 & & & \\
\hline Social & 0.634 & 0.684 & 1.000 & & \\
\hline Spiritual & 0.051 & 0.192 & 0.088 & 1.000 & \\
\hline Total score & 0.800 & 0.907 & 0.840 & 0.317 & 1.000 \\
\hline
\end{tabular}

KT-QoL, Korean version of the self-reported questionnaire for thyroid cancer patients.
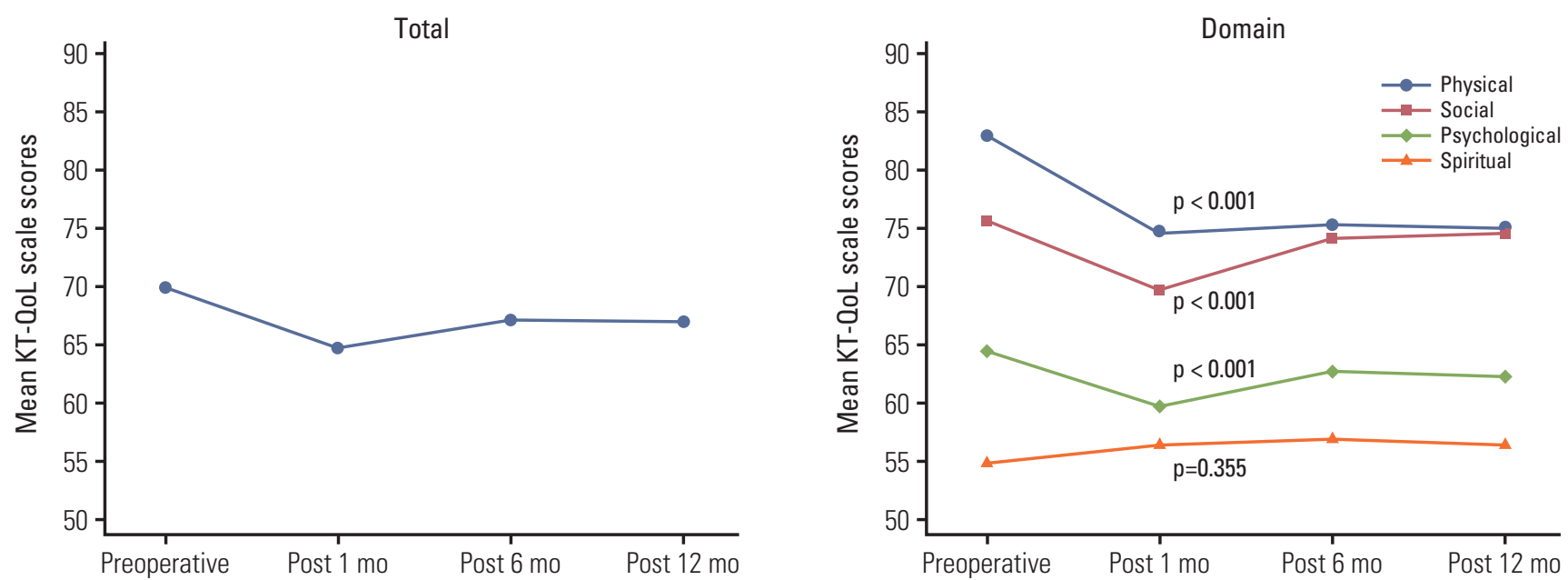

Fig. 1. Comparison of mean Korean version of the self-reported questionnaire for thyroid cancer patients (KT-QoL) scale scores over time. 

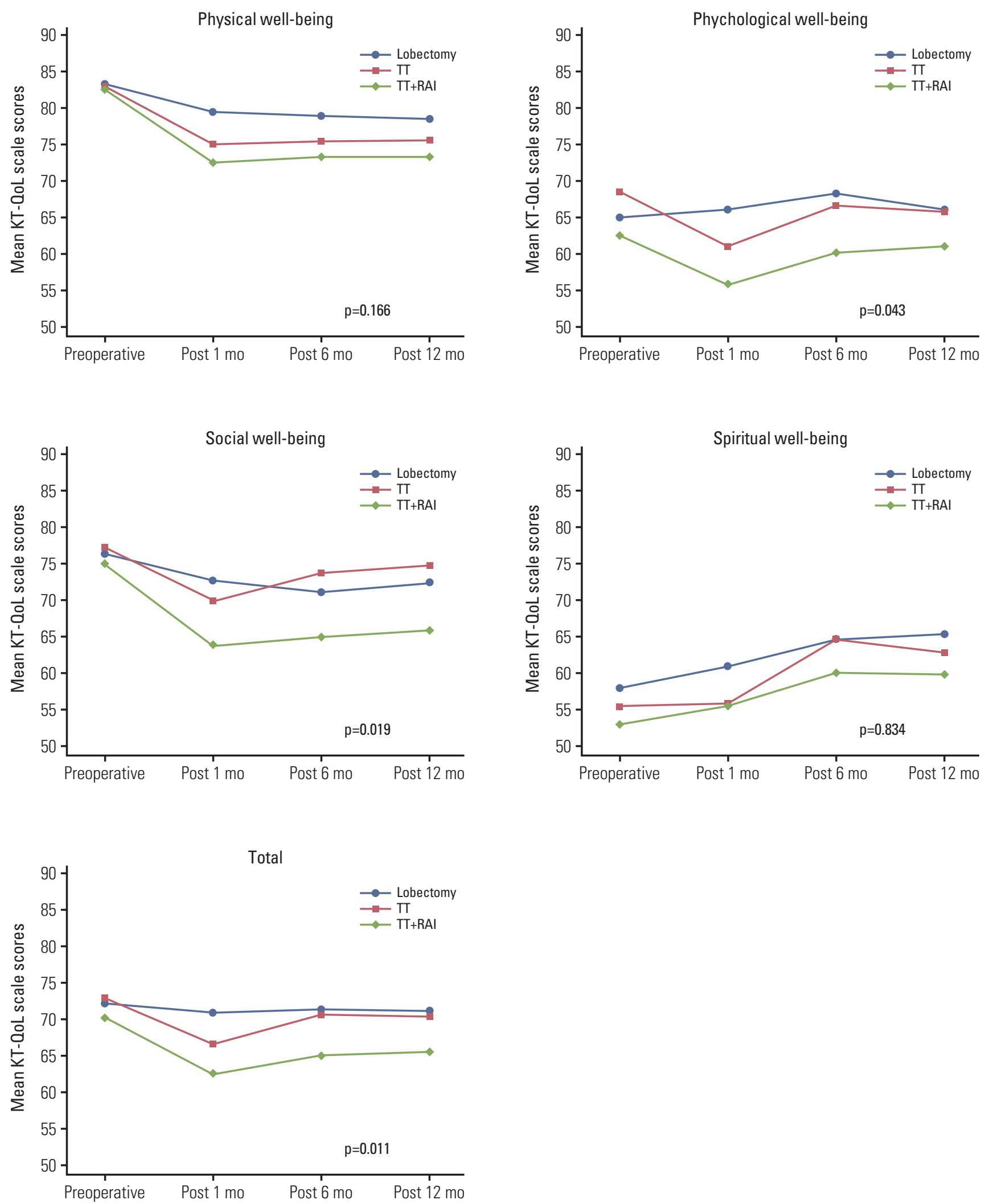

Fig. 2. Comparison of mean Korean version of the self-reported questionnaire for thyroid cancer patients (KT-QoL) scale scores over time according to treatment strategy. TT, total thyroidectomy; RAI, radioactive iodine. 

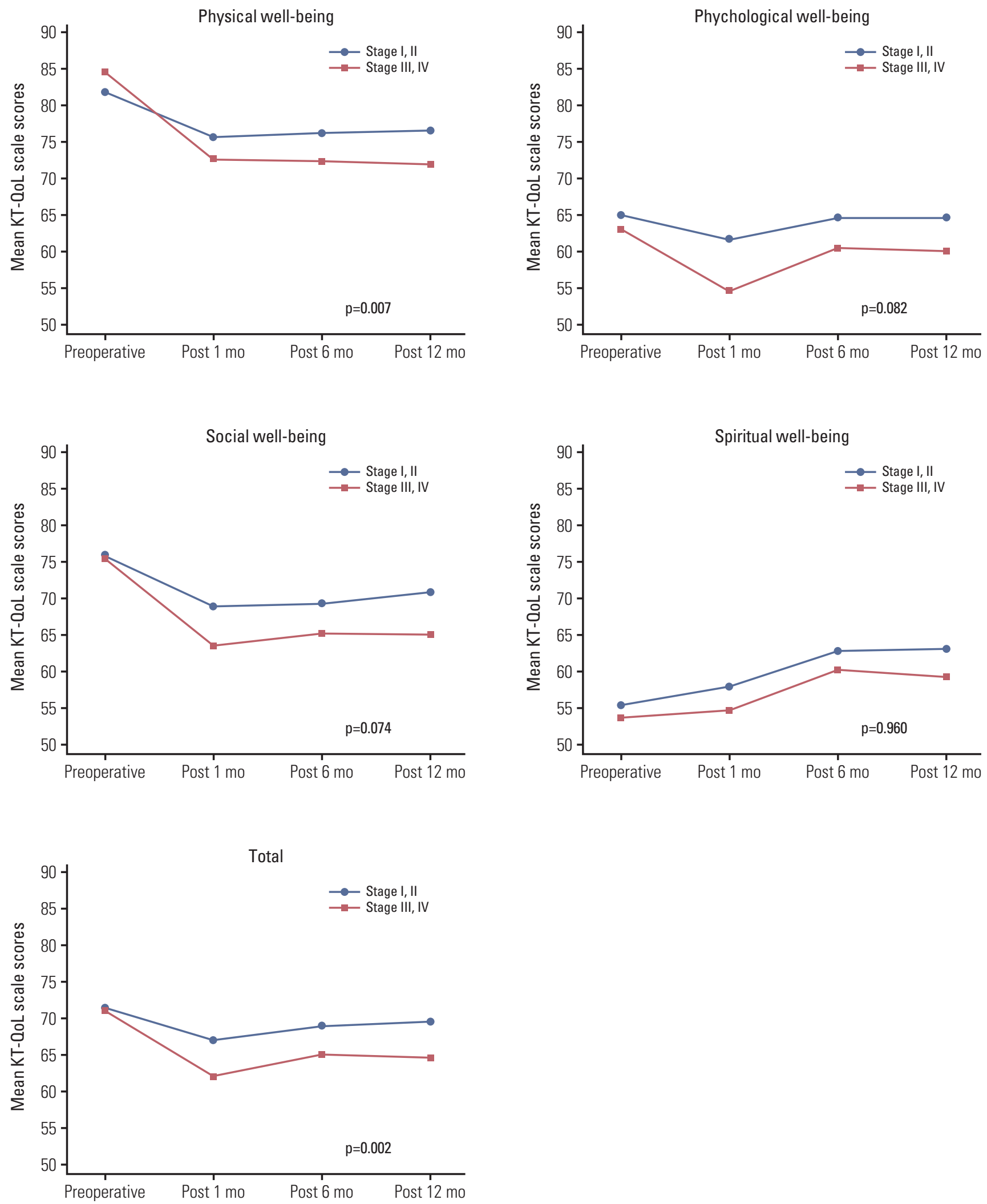

Fig. 3. Comparison of mean Korean version of the self-reported questionnaire for thyroid cancer patients (KT-QoL) scale scores over time according to tumor stages. 
groups. (Fig. 2).

When the QoL scores were compared by stages, the total QoL scores in advanced stages were much decreased during the postoperative periods compared to those in early stages $(\mathrm{p}=0.002)$ as expected. Also, the QoL scores for physical wellbeing $(p=0.007)$ were significantly decreased for those with advanced stages. Marginal significance was observed for the QoL scores for psychological ( $\mathrm{p}=0.082$ ) and social well-being $(p=0.074)$. However, the scores for spiritual well-being were not significantly different between stage groups (Fig. 3). No difference in QoL scores was observed when the scores were compared between male and female (data not shown).

\section{Discussion}

In this study, we developed the KT-QoL questionnaire and evaluated its reliability and validity in Korean patients in order to establish a system for better and more specific documentation of QoL during the treatment of thyroid cancer. Considering DTC has become the most prevalent cancer, and enhancing quality of survivorship is becoming a major issue for these patients, solid documentation of self-reported QoL might be important for precise monitoring of outcomes. The questionnaire system provided herein, designed specifically for thyroid cancer, could serve this role.

Our analysis revealed good internal validity of the KT-QoL. IICs were greater than 0.4 , and IDVs were greater than $85 \%$ in all time points. This implies that the items in the same dimension are well grouped, and that items in different dimensions are well discriminated. Internal reliability analysis with Cronbach's alpha $>0.9$ revealed an excellent internal reliability. Test-retest reliability analysis with IIC also showed good consistency at each time point. However, items 2, 17, and 27 showed low IIC values. Item 2 asks overall physical well-being, and items 17 and 27 ask about support and uncertainty, respectively. Because the original English version did not report any data on internal validity, we are not certain whether this is a consequence of cultural difference or a problem in the questionnaire itself. Our analysis revealed also good external validity. The Spearman's correlation between item $1 \mathrm{j}$ (voice changes) of KT-QoL and VHI30 at each time point showed moderate positive correlation at preoperative period, whereas strong correlation at postoperative 1, 6, and 12 months. This may be due to the voice change after surgery. In general, thyroid cancer does not produce any symptoms unless they invade adjacent structures including recurrent laryngeal nerve, trachea and esophagus. They usually have normal voice before surgery, however, experienced significant vocal compromise after surgery
$[15,19]$. Preoperative normal voice may show relative low correlation between $1 \mathrm{j}$ of KT-QoL and VHI-30. Despite these minor problems, the result generally represents good internal and external validity $[14,20]$.

Several well-known questionnaires measure the QoL in cancer patients, including the SF-36, EORTC-QLQ C30, and FACT-G questionnaires. These questionnaires focus on the general status of patients' QoL. Most QoL studies in thyroid cancer used these general questionnaires [10]. These general questionnaires are good not only to assess the general demand and deficiency of life quality, but also to compare relative life quality between different types of cancers. However, these may not be able to fully evaluate the impact of thyroid-specific treatment on the QoL. All patients after total thyroidectomy should take the thyroid hormone replacement, and some patients should undergo additional RAI therapies based on their disease status. Most patients present with minor or major voice problems after surgery [15]. All of these could have an influence on a given patient's QoL. The KT-QoL developed and validated in this study includes the symptoms of surgery related complications, symptoms of thyroid hormone excess and deficiency, as well as general symptoms. Thus, it can capture the problems that are specific to thyroid cancer patients. Moreover, it consists of 49 items (54 sub-scales), which are easy to understand and do not take long time to complete. This is helpful for patient compliance. In fact, among 272 eligible patients, only 17 patients (6.3\%) were excluded from the analysis due to missing responses. All these represent the suitability of the KT-QoL.

However, before using this questionnaire, the researchers should pay attention to one problem. This stems from the fact that this questionnaire includes all thyroid related symptoms, including treatment related symptoms. Item 12 is part of the psychological domain, and it consists of multiple subscale items that are focused on the psychological effect of surgery, RAI therapy, and thyroid hormone replacement therapy. These important items can measure the decrease in QoL after treatment. However, these cannot be evaluated before treatment. Therefore, caution is needed when interpreting the results of the treatment effect on QoL. If the researchers measure the QoL scores as a sum of each item score, the total QoL scores will decrease in patients who underwent surgery or RAI therapy. Therefore, we removed the item 12 in questionnaires for validation. We believe that item 12 should be analyzed separately, and that when doing so the reference baseline time should be immediately after treatment.

Our analysis using KT-QoL revealed that the patients experienced decreased QoL after thyroidectomy. After surgery, the physical, psychological, and social domains were decreased at 1 month after surgery, although they showed improvement 6 months after surgery. Postoperative 12 
months QoL scores were not changed significantly compared to 6 months after surgery. However, thyroidectomy did not seem to affect the spiritual aspect. The QoL scores for spiritual domain did not change significantly along the postoperative periods. On correlation analysis between each domain, the spiritual domain also showed low correlation with other domains. This is in line with QoL studies using original T-QoL. They also reported that the scores for spiritual domain did not show any significant change after thyroidectomy [14]. Most of QoL questionnaires including EORTC-QLO C30, SF-36, and FACT-G did not weight to the spiritual domains [11-13]. Therefore, the impact of thyroid cancer on the spiritual domain is not well-known. One of the possible explanations is that the treatment of thyroid cancer is known to be relatively easy to endure compared to that of other types of cancer, and, therefore, it is not a major determinant of the change in spiritual behavior. Other possible explanation is that low enrolled patients fail to document significant change about the spiritual aspect. In our serial analysis, the QoL scores for spiritual domain tends to increase after thyroidectomy, which may mean that the patients have serious attitude toward life after being diagnosed with thyroid cancer. This hypothesis should be validated with larger patients' series.

Surgical extent and postoperative RAI administration have impacts on the patient's QoL, mainly in social and psychological aspects. The QoL scores in patents with lobectomy did not change after surgery, however, total thyroidectomy affected patients' QoL. Patients with total thyroidectomy groups experienced decreased QoL at postoperative 1 month. These scores were recovered at postoperative 6 months and 12 months in patients with total thyroidectomy who did not receive RAI. However, patients receiving RAI continued to experience decreased QoL within 1 year after thyroidectomy. Total thyroidectomy tends to present with more surgery-related complications despite the obvious benefit for recurrence and survival [10,21]. Recently developed thyroidectomy guidelines reflect these aspects and recommend the decreased surgical extent and RAI therapy [2].

The advanced stages also have impact on patients' QoL. The patients with advanced stages experienced decreased total QoL scores during the postoperative periods. This may be partly due to the difference of treatment modality between stages. In our data set, $88.1 \%$ of lobectomy and $89.5 \%$ of total thyroidectomy without RAI thearpy patients had early stages. For proper comparison, the surgical extent and subsequent RAI administration should be adjusted, and a controlled study with a large sample size is required.

In summary, questionnaires must be validated when developed in a different language for appropriate use. In this study, we developed and validated KT-QoL. Using KT-QoL, we found that patients' QoL decreased after surgery. The treating physician should be mindful of this to improve postsurgery patient care.

\section{Electronic Supplementary Material}

Supplementary materials are available at Cancer Research and Treatment website (http:// www.e-crt.org).

\section{Conflicts of Interest}

Conflict of interest relevant to this article was not reported.

\section{Acknowledgments}

This research was supported by the Program through the National Cancer Center of Korea funded by the Ministry of Science, ICT and Future planning (NCC-1410920-3) and a grant from the National Cancer Center of Korea (NCC-1710440-1).

\section{References}

1. Siegel R, Naishadham D, Jemal A. Cancer statistics, 2013. CA Cancer J Clin. 2013;63:11-30.

2. Haugen BR, Alexander EK, Bible KC, Doherty GM, Mandel SJ, Nikiforov YE, et al. 2015 American Thyroid Association management guidelines for adult patients with thyroid nodules and differentiated thyroid cancer: The American Thyroid Association Guidelines Task Force on Thyroid Nodules and Differentiated Thyroid Cancer. Thyroid. 2016;26:1-133.

3. Perros P, Boelaert K, Colley S, Evans C, Evans RM, Gerrard $\mathrm{GE}$, et al. Guidelines for the management of thyroid cancer. Clin Endocrinol (Oxf). 2014;81 Suppl 1:1-122.

4. Verburg FA, Mader U, Tanase K, Thies ED, Diessl S, Buck AK, et al. Life expectancy is reduced in differentiated thyroid cancer patients $\geq 45$ years old with extensive local tumor invasion, lateral lymph node, or distant metastases at diagnosis and normal in all other DTC patients. J Clin Endocrinol Metab. 2013;98:172-80.

5. Maier TM, Schober O, Gerss J, Gorlich D, Wenning C, Schaefers $M$, et al. Differentiated thyroid cancer patients more than 60 years old paradoxically show an increased life expectancy. J Nucl Med. 2015;56:190-5.

6. Lang BH, Wong CK, Tsang JS, Wong KP. A systematic review and meta-analysis comparing outcomes between roboticassisted thyroidectomy and non-robotic endoscopic thyroidec- 
tomy. J Surg Res. 2014;191:389-98.

7. Randolph GW, Dralle H; International Intraoperative Monitoring Study Group, Abdullah H, Barczynski M, Bellantone R, et al. Electrophysiologic recurrent laryngeal nerve monitoring during thyroid and parathyroid surgery: international standards guideline statement. Laryngoscope. 2011;121 Suppl 1: S1-16.

8. Van Nostrand D. The benefits and risks of I-131 therapy in patients with well-differentiated thyroid cancer. Thyroid. 2009;19:1381-91.

9. Bilimoria KY, Bentrem DJ, Ko CY, Stewart AK, Winchester DP, Talamonti MS, et al. Extent of surgery affects survival for papillary thyroid cancer. Ann Surg. 2007;246:375-81.

10. Husson O, Haak HR, Oranje WA, Mols F, Reemst PH, van de Poll-Franse LV. Health-related quality of life among thyroid cancer survivors: a systematic review. Clin Endocrinol (Oxf). 2011;75:544-54.

11. Han CW, Lee EJ, Iwaya T, Kataoka H, Kohzuki M. Development of the Korean version of Short-Form 36-Item Health Survey: health related QOL of healthy elderly people and elderly patients in Korea. Tohoku J Exp Med. 2004;203:189-94.

12. Yun YH, Park YS, Lee ES, Bang SM, Heo DS, Park SY, et al. Validation of the Korean version of the EORTC QLQ-C30. Qual Life Res. 2004;13:863-8.

13. Lee EH, Chun M, Kang S, Lee HJ. Validation of the Functional Assessment of Cancer Therapy-General (FACT-G) scale for measuring the health-related quality of life in Korean women with breast cancer. Jpn J Clin Oncol. 2004;34:393-9.

14. Dow KH, Ferrell BR, Anello C. Quality-of-life changes in patients with thyroid cancer after withdrawal of thyroid hor- mone therapy. Thyroid. 1997;7:613-9.

15. Ryu J, Ryu YM, Jung YS, Kim SJ, Lee YJ, Lee EK, et al. Extent of thyroidectomy affects vocal and throat functions: a prospective observational study of lobectomy versus total thyroidectomy. Surgery. 2013;154:611-20.

16. American Thyroid Association (ATA) Guidelines Taskforce on Thyroid Nodules and Differentiated Thyroid Cancer, Cooper DS, Doherty GM, Haugen BR, Kloos RT, Lee SL, et al. Revised American Thyroid Association management guidelines for patients with thyroid nodules and differentiated thyroid cancer. Thyroid. 2009;19:1167-214.

17. Fayers PM, Machin D. Quality of life: the assessment, analysis and reporting of patient-reported outcomes. 3rd ed. New Jersey, NJ: Wiley Blackwell; 2015. p. 393-427.

18. Ware JE Jr, Gandek B. Methods for testing data quality, scaling assumptions, and reliability: the IQOLA Project approach. International Quality of Life Assessment. J Clin Epidemiol. 1998;51:945-52.

19. Ryu CH, Ryu J, Ryu YM, Lee YJ, Lee EK, Kim SK, et al. Administration of radioactive iodine therapy within 1 year after total thyroidectomy does not affect vocal function. J Nucl Med. 2015;56:1480-6.

20. Quality of Life: thyroid version [Internet]. Duarte, CA: City of Hope National Medical Center and Beckman Research Institute; 2000 [cited 2016 Dec 28]. Available from: http://prc.coh. org/QOL-Thy.pdf.

21. Hauch A, Al-Qurayshi Z, Randolph G, Kandil E. Total thyroidectomy is associated with increased risk of complications for low- and high-volume surgeons. Ann Surg Oncol. 2014;21: 3844-52. 